\title{
A NOTE ON PIERCING A DISK
}

\section{P. H. DOYLE AND J. G. HOCKING}

In this note we give a sufficient condition that a 2-cell or disk in $E^{3}$ be pierced by a tame arc at each point of its interior. This condition is simply that each arc in the disk be tame. The method of proof leans heavily upon some results of Bing and Moise as well as upon a theorem on the union of tame disks by one of the present authors.

REMARK. Lemma 7 of [2] asserts that if two tame disks in $E^{3}$ intersect in a simple arc on the boundary of each, then the union of the two disks is a tame disk. The proof of this result is an application of Theorem 5.1 of [4]. It is then a simple matter to see that if $D$ is a disk in $E^{3}$ having an interior which is triangulated in a locally finite manner into tame 2-cells, then $D$ is locally tame at each of its interior points. If in addition $D$ has a tame boundary, then $D$ is tame by either Theorem 5.13 of [5] or Theorem 5.1 of [4]. These observations will be used in our proof.

Theorem. Let $D$ be a disk in $E^{3}$ such that each arc in $D$ is tame. Then $D$ is pierced by a tame arc at each point of $D^{0}$ (interior of $D$ ).

Proof. Let $q$ be any point in $D^{0}$. Let $R$ be the plane rectangle $\{(x, y) \mid-1 \leqq x \leqq 1,-1 \leqq y \leqq 1\}$ and let $h$ be a homeomorphism of $R$ onto $D$. There is no loss of generality in assuming that $h^{-1}(q)$ $=(0,0)$. We construct an infinite cell decomposition of $R$ by means of the line segments

$$
\begin{aligned}
J_{0} & =\{(x, y) \mid x=0,-1 \leqq y \leqq-1, \\
K_{0} & =\{(x, y) \mid 0 \leqq x \leqq 1, y=0\}, \\
J_{n}^{-} & =\{(x, y) \mid x=1 / n,-1 \leqq y \leqq 1 ; n>1\}, \\
J_{n}^{+} & =\{(x, y) \mid x=(n-1) / n,-1 \leqq y \leqq 1 ; n>2\}, \\
K_{n}^{-} & =\left\{(x, y) \mid 0 \leqq x \leqq 1, y=-1 / 2-(-1)^{n}(n-1) / 2 n ; n>1\right\}, \\
K_{n}^{+} & =\left\{(x, y) \mid 0 \leqq x \leqq 1, y=1 / 2-(-1)^{n}(n-1) / 2 n ; n>1\right\} .
\end{aligned}
$$

Denote the union of all of these segments together with the closure of this union by $G^{\prime}$. The grid $G^{\prime}$ decomposes $R$ into countably many closed disks and an isomorphic decomposition of $D$ is induced through the homeomorphism $h$.

Let $R_{1}=\{(x, y) \mid 0 \leqq x \leqq 1,-1 \leqq y \leqq 1\}$ and $R_{2}=\bar{R}-\left(R_{1}-J_{0}\right)$. Denote $h\left(R_{i}\right)$ by $D_{i}, i=1,2$, and $h\left(G^{\prime}\right)$ by $G$. (Clearly, each arc in $G$ is

Received by the editors November 20, 1958. 
tame by our assumption on $D$.) Define the real-valued function $f(x)$ on $D_{1}$ by setting $f(x)=2^{-1} \rho_{1}(x)$ where $\rho_{1}(x)$ is the distance from the point $x$ to the compact set $G \cup D_{2}$. Using the Bing Approximation Theorem [1], we choose a homeomorphism $g$ of $D_{1}$ into $E^{3}$ such that (i) $g\left(D_{1}\right)$ is locally polyhedral except on $G$ and (ii) $\rho[x, g(x)] \leqq f(x)$. Letting $g\left(D_{1}\right)=D_{1}^{\prime}$, we observe that repeated application of the Remark above together with the construction of $G$ implies that $D_{1}^{\prime}$ has a tame boundary and is locally tame in its interior. Hence in view of the Remark, $D_{1}^{\prime}$ is tame.

We next "swell" $D_{1}^{\prime}$ into a tame 3 -cell $e^{3}$ which is locally polyhedral except on the boundary of $D_{1}$ and which is such that $\left(D_{1}^{\prime}\right)^{0}$ lies in $\left(e^{3}\right)^{0}$ while $e^{3} \cap D_{2}$ is precisely the tame arc $h\left(J_{0}\right)$. This is done by making use of Lemma 5.1 of [3] and Theorem 9.3 of [4]. We denote the boundary of $e^{3}$ by $S$ and observe that the boundary of $D_{1}$ separates $S$ into two hemispheres, say $S_{1}$ and $S_{2}$.

Let $\left\{C_{i}\right\}$ be a sequence of all disks in the decomposition of $D_{1}$ by $G$ for which $S_{1} \cap C_{i}$ is not empty. Each intersection $S_{1} \cap C_{i}$ is a compact set in the interior $C_{i}^{0}$ of $C_{i}$ because the hemisphere $S_{1}$ is disjoint from the grid $G$ except along the boundary of $D_{1}$. Thus, for each $i$, the distance $\rho\left(S_{1} \cap C_{i}, S_{1} \cap D_{1}-C_{i}\right)$ is positive. Appealing to the Zoretti Theorem [6] we note that each component $K_{i \alpha}$ of $S_{1} \cap C_{i}$ may be enclosed in a simple closed curve $A_{i \alpha}$ in $S_{1}^{0}$ with the property that $A_{i \alpha} \cap\left(S_{1} \cap C_{i}\right)$ is empty and $A_{i \alpha}$ is as close to $K_{i \alpha}$ as we may wish. There is no loss of generality in assuming $A_{i \alpha}$ to be polygonal. Hence for each $i$ we may choose a finite number of the curves $A_{i \alpha}$ and so obtain a finite number of polyhedral disks $C_{i j}^{\prime}$ in $S_{1}^{0}$ whose interiors cover $S_{1} \cap C_{i}$.

To give the desired construction, we choose the finite number of simple closed curves $A_{1 j}$ in such a way that $\rho\left(A_{1 j}, S_{1} \cap C_{1}\right)$ $<2^{-1} \rho\left(S_{1} \cap C_{1}, S_{1} \cap D_{1}-C_{1}\right)$. Let the union of the corresponding disks $C_{1 j}^{\prime}$ be the polyhedral set $C_{1}^{\prime}$. Then we choose the curves $A_{2 j}$ in such a way that $\rho\left(A_{2 j}, S_{1} \cap C_{2}\right)$ is less than one half of the minimum of $\rho\left(S_{1} \cap C_{2}, S_{1} \cap D_{1}-C_{2}\right)$ and $\rho\left(C_{1}^{\prime}, S_{1} \cap D_{1}-C_{1}\right)$. In this way we obtain a polyhedral set $C_{2}^{\prime}$ in $S_{1}^{0}$ such that $C_{1}^{\prime} \cap C_{2}^{\prime}$ and $C_{2}^{\prime} \cap \bar{D}_{1}-\left(C_{2}-J_{0}\right)$ are both empty. Continuing this procedure in the obvious way, we obtain a sequence $\left\{C_{i}^{\prime}\right\}$ of disjoint closed polyhedral sets in $S_{1}^{0}$ such that the union $\mathrm{U}\left(C_{i}^{\prime}\right)^{0}$ contains $S_{1}^{0} \cap D_{1}$. By simply ordering the components of each set $C_{i}^{\prime}$ we obtain a sequence $\left\{C_{i}^{\prime \prime}\right\}$ of connected closed disjoint polyhedral sets in $S_{1}^{0}$ covering $S_{1}^{0} \cap D_{1}$. We note that the method of construction implies that the diameters of the sets $C_{i}^{\prime \prime}$ approach zero as $i$ increases indefinitely.

Once again the Zoretti Theorem may be applied to enclose each 
set $C_{i}^{\prime \prime}$ in a simple closed curve $A_{i}$ in $S_{1}^{0}$, each point of $A_{i}$ being as close to $C_{i}^{\prime \prime}$ as desired. Again we assume each $A_{i}$ to be polygonal. A construction almost identical to that above may be described inductively so as to yield a sequence of polygonal simple closed curves $\left\{A_{i}\right\}$ such that $A_{i} \cap A_{j}$ is empty if $i \neq j$, each $A_{i}$ bounds a polyhedral $\operatorname{disk} P_{i}$ in $S_{1}^{0}$ with $C_{i}^{\prime \prime}$ lying in $P_{i}^{0}$ and as $i$ increases without bound, the diameter of $P_{i}$ approaches zero. However it may happen in this construction that for $i \neq j$ we have $P_{i}$ contained in $P_{j}^{0}$. So we let $\left\{P_{j}^{\prime}\right\}$ be a subsequence selected from $\left\{P_{i}\right\}$ such that $P_{j}^{\prime} \cap P_{k}^{\prime}$ is empty if $j \neq k$ and such that for each $i$ there is a $j$ such that $P_{i}$ lies in $P_{j}^{\prime}$. Clearly we still have the fact that $\mathrm{U}\left(S_{1}^{0} \cap C_{i}\right)$ is contained in $U P_{j}^{\prime}$.

Let $x$ be any point in $S_{1}^{0}-U P_{j}^{\prime}$. Since $S_{1}$ is polyhedral except on its boundary, there is a tame arc $T$ in $S_{1}$ with endpoints $x$ and $q$ and such that $T-q$ lies in $S_{1}^{0}$. We may choose $T$ to be polygonal except at $q$. In general $T$ will intersect some of the disks in the sequence $\left\{P_{j}^{\prime}\right\}$. We construct an arc $T_{\infty}$ having the same properties as $T$ but which does not meet the interior of any disk $P_{j}^{\prime}$. To do this, we describe a sequence of $\operatorname{arcs} T_{i}$ as follows: If $T$ fails to meet the interior of $P_{1}^{\prime}$, we let $T_{1}=T$. Otherwise, let $T$ be parametrized by way of a homeomorphism $p$ of the unit interval where $p(0)=x$ and $p(1)=q$. There will be a first and a last point in which $T$ intersects $P_{1}^{\prime}$. We replace the corresponding subarc on $T$ by the shorter of the two arcs on the boundary $A_{1}^{\prime}$ of $P_{1}^{\prime}$ having the same endpoints and call the resulting arc $T_{1}$. Suppose that $T_{j}$ has been defined so that it is an arc from $x$ to $q$, that $T_{j}-q$ lies in $S_{1}^{0}$ and $T_{j} \cap \bigcup_{n=1}^{j}\left(P_{n}^{\prime}\right)^{0}$ is empty. The arc $T_{j+1}$ is $T_{j}$ if $T_{j} \cap\left(P_{n+1}^{\prime}\right)^{0}$ is empty; otherwise we proceed as was done in obtaining $T_{1}$ from $T$. That is, $T_{j}$ is parametrized and a subarc of $T_{j}$ is replaced by an arc in $A_{j+1}^{\prime}$, the boundary of $P_{j+1}^{\prime}$. It is easily shown that the sequence of arcs $\left\{T_{j}\right\}$ converges to an arc $T_{\infty}$ since the diameters of the disks $P_{j}^{\prime}$ approach zero with increasing $j$. By construction $T_{\infty}$ is disjoint from the sets $C_{i}^{\prime \prime}$ and lies in $S_{1}^{0}$ except at $q$. It follows that $T_{\infty}$ meets the disk $D_{1}$ only at $q$. Also since $S_{1}$ is disjoint from $D_{2}$ except along the arc $h\left(J_{0}\right)$ in the boundary of $S_{1}, T_{\infty}$ is disjoint from the disk $D_{2}$ except at the point $q$.

By an identical construction an arc $T_{\infty}^{\prime}$ can be obtained on the other hemisphere $S_{2}$ such that $T_{\infty}^{\prime} \cap D=q$. The union $A=T_{\infty} \cup T_{\infty}^{\prime}$ is a tame arc since it lies on a tame 2-sphere $S$ and $A$ meets $D$ only at $q$. Thus $A$ pierces $D$ at $q$ and the proof is complete.

The following results are now obvious.

CoRollary 1. If a disk $D$ in $E^{3}$ has the property that each arc in $D^{0}$ is tame, then $D$ may be pierced by a tame arc at each point of $D^{0}$. 
Corollary 2. $A$ disk in $E^{3}$ which is pierced by no tame arc has a wild arc in each of its open sets.

We believe the converse of Corollary 1 to be true also but have not been able to prove it. In this connection we may call attention to a conjecture of $\mathrm{O}$. G. Harrold which asserts that if $D$ is a disk in $E^{3}$ such that all arcs in $D$ are tame, then $D$ itself is tame. Our result lends some support to this conjecture.

\section{REFERENCES}

1. R. H. Bing, Approximating surfaces with polyhedral ones, Ann. of Math. vol. 65 (1957) pp. 456-483.

2. P. H. Doyle, Tame, finite complexes in three-space, Thesis, University of Tennessee, 1957.

3. O. G. Harrold, E. E. Posey and H. C. Griffith, A characterization of tame curves in three-space, Trans. Amer. Math. Soc. vol. 79 (1955) pp. 12-34.

4. E. E. Moise, Affine structures in 3-manifolds, VIII, Invariance of the knot-types; Local tame imbedding, Ann. of Math. vol. 59 (1954) pp. 159-170.

5. E. E. Posey, Almost polyhedral cells in euclidean space, Thesis, University of Tennessee, 1954.

6. G. T. Whyburn, Topological analysis, Princeton University Press, 1958.

Michigan State University 